\title{
1 Immunotherapy efficacy in colorectal cancer is dependent on activation of
}

3 Lukas F. Mager ${ }^{1, *}$, Regula Burkhard ${ }^{2}$, Noah C.A. Cooke ${ }^{1}$, Kirsty Brown ${ }^{1}$, Hena Ramay ${ }^{3}$,

4 Seungil Paik ${ }^{4}$, John Stagg ${ }^{5}$, Ryan A. Groves ${ }^{6}$, Marco Gallo ${ }^{4}$, Ian A. Lewis ${ }^{6}$, Markus B.

5 Geuking $^{2}$, Kathy D. McCoy ${ }^{1,7, *}$

6

$7 \quad{ }^{1}$ Dept. of Physiology and Pharmacology, Snyder Institute of Chronic Diseases, Cumming

8 School of Medicine, University of Calgary, Calgary, Canada

$9 \quad{ }^{2}$ Dept. of Microbiology, Immunology and Infectious Diseases, Snyder Institute of Chronic

10 Diseases, Cumming School of Medicine, University of Calgary, Calgary, Canada

$11{ }^{3}$ International Microbiome Centre, Cumming School of Medicine, University of Calgary,

12 Canada

$13{ }^{4}$ Departments of Biochemistry and Molecular Biology and Physiology and Pharmacology,

14 Charbonneau Cancer Institute, Alberta Children's Hospital Research Institute, Cumming

15 School of Medicine, University of Calgary, Canada

${ }^{5}$ Centre de Recherche du Centre Hospitalier de l'Université de Montréal et Institut du Cancer de Montréal, Québec, Canada

${ }^{6}$ Dept. of Biological Sciences, University of Calgary, Calgary, Canada

*Corresponding authors: Kathy D. McCoy and Lukas F. Mager.

e-mail: kathy.mccoy@ucalgary.ca, lukas.mager@ucalgary.ca 


\section{Summary}

28 Cancer is a leading cause of death globally. Checkpoint blockade therapies offer a promising treatment for many cancers, but have been ineffective for colorectal cancers. Previous studies have shown a dependency of immunotherapies on the microbiota. Consequently, we

31 hypothesized that specific gut bacteria promote immunotherapy for colorectal cancer. We 32 identify three commensal bacteria and a microbial metabolite, inosine, that enhance the 33 efficacy of immune checkpoint blockade therapy in colorectal cancer. We show that inosine interacts with the adenosine $\mathrm{A}_{2 \mathrm{~A}}$ receptor on $\mathrm{T}$ cells resulting in intestinal $\mathrm{Th} 1$ cell differentiation. Decreased gut barrier function induced by immunotherapy increased the translocation of bacterial metabolites and promoted cancer protective Th1 cell activation. This microbial-metabolite-immune circuit provides a mechanism for a new class of bacteriaenhanced checkpoint blockade therapies. The efficacy of this mechanism differs among colorectal cancer subtypes and highlights the strengths as well as potential limitations of this novel bacterial co-therapy for cancer.

\section{Keywords}

42 Cancer, Immunotherapy, Microbiome

\section{Introduction}

44 Colorectal cancer (CRC) is the second and third most common malignancy in Western countries in women and men, respectively (Ferlay et al., 2015). In addition to genetic aberrations, which are essential for the development of CRC, other disease-contributing factors have been identified. These include the microbiota and inflammation, whereby inflammation can drive or inhibit CRC development. Interferon (IFN)- $\gamma$ producing T helper type 1 (Th1) cells are known to be protective (Mager et al., 2016; Mlecnik et al., 2016; Wang et al., 2015), whereas interleukin (IL)-17-producing Th17 cells promote CRC development (Galon et al., 
2006; Grivennikov et al., 2012; Le Gouvello et al., 2008). In fact, the impact of the immune system is so potent that immune cell infiltration in the tumor is a superior prognostic factor compared to the classical tumor-lymph nodes-metastasis (TNM) system in CRC (Anitei et al., 2014; Mlecnik et al., 2016). Similarly, the microbiota also impacts on CRC progression(Arthur et al., 2012; Dejea et al., 2018) and may even alter the efficacy of chemotherapeutics (Iida et al., 2013; Viaud et al., 2013).

Immune checkpoint blockade (ICB) therapy is an efficient anti-cancer strategy that utilizes the therapeutic potential of the immune system. Most notably, ICB inhibitors targeting cytotoxic T-lymphocyte-associated antigen 4 (CTLA-4), programmed cell death protein 1 (PD-1), or its ligand (PD-L1) have shown great success in the treatment of various cancers, including melanoma, renal cell carcinoma, and non-small cell lung cancer (Brahmer et al., 2012; Hodi et al., 2010). More recently, seminal work has shown that the efficacy of ICB therapy is dependent Vetizou et al., 2015).

Despite these exciting advances, ICB therapy efficacy in CRC has been disappointing 66 (Brahmer et al., 2012), with only 5-10\% of all CRC patients responding (Le et al., 2017). Moreover, the detailed molecular mechanisms through which bacteria enhance the efficacy of ICB therapies remains unclear. Here, we identified three bacterial species that promote ICB efficacy in CRC and identified inosine as a critical bacterial metabolite that promoted differentiation of Th1-mediated anti-tumor immunity.

\section{$71 \quad$ Results}

\section{ICB therapy efficacy depends on the gut microbiota}

73 We first questioned whether ICB therapy efficacy in CRC is dependent on the microbiota.

74 Heterotopic MC38 colorectal cancers were implanted into germ-free (GF) and specific 
pathogen free (SPF) mice and, upon palpable tumor development, ICB therapy was initiated,

which led to smaller tumors in SPF animals (Figure S1A and B). Moreover, intratumoral and splenic $\mathrm{CD}^{+}$and $\mathrm{CD}^{+} \mathrm{T}$ cell activation and proliferation were markedly increased in SPF animals (Figure S1C - N). To ensure this was not merely a reflection of the immature immune system of GF mice, we also assessed the effect and ICB therapy in antibiotic-treated SPF mice (Figure S1O). Compared to control treatment, broad spectrum antibiotics also reduced ICB therapy efficacy in tumor-bearing SPF mice (Figure S1P - T). These results indicated that ICB efficacy is enhanced in the presence of microbes, corroborating previous reports with other tumor types (Vetizou et al., 2015).

\section{Identification of ICB-promoting bacteria in CRC}

Clinically, ICB therapies are notoriously ineffective in most CRC cases (Le et al., 2015) and heterotopic tumors may not adequately model the spatially close interactions between the gut microbiota and local immunity in intestinal tumors. We therefore employed a more physiological model of CRC to investigate the interactions between the microbiota and immunity in the context of ICB therapy. Intestinal tumors were induced using azoxymethane (AOM) and dextran sulfate sodium (DSS) in SPF animals. Following tumor development, we evaluated the ability of ICB therapy to induce anti-tumor immunity (Figure 1A). Notably, ICB therapy led to smaller and fewer tumors (Figure 1B and C), reduced cancer stem cell numbers (Figure 1D), increased immune cell infiltration into the tumors (Figure 1E), and increased $\mathrm{CD}^{+} \mathrm{T}$ cell frequencies in the tumor draining lymph node together with increased splenic $\mathrm{CD}^{+}$and $\mathrm{CD}^{+} \mathrm{T}$ cell activation (Figure $1 \mathrm{~F}-\mathrm{H}$ ). In this model, anti-CTLA-4 tumoricidal effects were greater than those induced by anti-PD-L1 treatment when using the same antibody dose. In order to identify potentially beneficial tumor-associated bacteria, we performed $16 \mathrm{~S}$ rRNA gene V4 region amplicon sequencing of genomic DNA isolated from homogenized tumors as well as anaerobic culture of homogenized tumor tissue. Microbial sequencing 
revealed that the tumor-associated bacterial community composition of ICB-treated tumors differed from that of control-treated tumors (Figure S2A and Figure 1I). Furthermore, we were these cultured bacteria were found only in the ICB-treated group, whereas four were found only in the control group (Figure 1J).

Interestingly, Akkermansia muciniphilia, which was recently identified to enhance the efficacy of anti-PD-L1 and anti-PD-1 treatments in lung and kidney cancers (Routy et al., 2018), was one of the seven bacteria cultured only from ICB-treated tumors. We also performed 16S rRNA gene V4 amplicon sequencing of fecal samples from control and ICB groups but found no significant differences in microbiota composition (Figure S2B, S2C and Table S1), indicating that the tumor-associated bacterial communities provided a better source for identification of

111 ICB-promoting bacteria in CRC.

112 To address whether the bacteria that were found to be enriched in the ICB-treated tumors were

113 able to boost the efficacy of ICB therapy, we selected five of the isolated culturable bacterial

114 species for monocolonization of GF mice. Monocolonized or GF mice were injected with

115 MC38 tumor cells, treated with anti-CTLA-4 upon palpable tumor development and assessed

116 for effects on tumor growth and anti-tumor immunity (Figure 2A). We chose the heterotopic

117 model of CRC for this approach as the development of orthotopic CRC is severely reduced in

118 animals with a limited microbiota (Schwabe and Jobin, 2013). Of the five bacteria tested,

119 monocolonization with Bifidobacterium pseudolongum (B.p.), Lactobacillus johnsonii (L.j.),

120 and Olsenella sp. (O.sp.) significantly enhanced the efficacy of anti-CTLA-4 treatment compared to GF mice or mice monocolonized with Colidextrbacter sp. (C.sp.) or Prevotella sp. (P.sp.) (Figure 2B-E). In addition, $\mathrm{CD} 4^{+}$and $\mathrm{CD} 8^{+} \mathrm{T}$ cell activation and proliferation were substantially increased in the tumors of B.p., L.j., and O.sp. monocolonized animals (Figure

124 2F-I). The isolated ICB-promoting B.p. strain also improved the efficacy of anti-PD-L1 
treatment in the MC38 heterotopic tumor model compared to the C.sp. control strain (Figure

S3), albeit to a lower extent than observed for anti-CTLA-4 treatment (at the same dose), which

is similar to our observations in the AOM/DSS model. Due to its greater observed efficacy, we

performed all subsequent mechanistic studies using anti-CTLA-4 treatment. Of note, antitumor immunity was dependent on anti-CTLA-4 or anti-PD-L1 co-therapy as monocolonization with B.p. alone was not able to reduce tumor growth (Figure S4A-D) or

131 induce anti-tumor immunity (Figure S4E-J), similar to previous studies with other ICB-

132 promoting bacteria(Routy et al., 2018; Vetizou et al., 2015).

\section{Induction of Th1 immunity through ICB promoting bacteria}

134 To investigate the mechanism by which the identified bacteria enhanced ICB therapy we selected B.p. as a representative of the beneficial bacteria since it appeared to have the strongest ICB-promoting effect. GF or C.sp. monocolonized served as negative controls. Previous studies revealed the ability of some bacteria to accumulate in the tumor environment where they locally stimulate the immune system and kill tumor cells through toxic metabolites (Zheng et al., 2018). Although bacteria were abundantly present in the feces of B.p. and C.sp. monocolonized mice, we were unable to detect bacteria or amplify 16S rDNA from heterotopic tumors of these mice (Figure S5), indicating that the beneficial effect of bacteria in this model

142 does not require bacteria to reside within the tumor itself. Compared to GF or C.sp.

143 monocolonized mice, B.p. monocolonization induced a significant increase in expression of the Th1 master transcription regulator T-bet in small intestinal lamina propria $\mathrm{CD} 4^{+} \mathrm{T}$ cells.

145 Similarly, albeit to a lower extent, B.p. induced T-bet expression in $\mathrm{CD}^{+} \mathrm{T}$ cells in the 146 mesenteric lymph nodes (MLN), but not in the spleen (Figure 3A-G). Intriguingly, B.p. did not 147 activate the effector function of Th1 cells as T-bet ${ }^{+-} \mathrm{IFN}-\gamma^{+}$double positive cells did not differ

148 between B.p, C.sp. or GF groups in any of the tissues assessed. Taken together, in the absence 149 of tumors and ICB therapy, B.p. alone promoted Th1 transcriptional differentiation without 
increasing effector function locally in the gut and draining lymph nodes but not systemically.

151 While B.p. had no effect on other $\mathrm{CD}^{+} \mathrm{T}$ cell subsets in the small intestine, it also increased

152 CD8 ${ }^{+}$T-bet $^{+}$T cells (Figure S6A-S6E). Moreover, B.p. had minimal impact on Th17 and Treg

153 cells in the MLN and spleen (Figure S6F-S6O).

\section{Systemic effect of ICB promoting bacteria}

Since B.p. alone promoted only local and not systemic Th1 differentiation during homeostasis,

(in the absence of a tumor) would induce systemic Th1 activation. Indeed, when combined with anti-CTLA-4, B.p. was able to significantly enhance splenic Th1 cell activation and effector function as evidenced by IFN- $\gamma$ production compared to C.sp. monocolonized or GF animals (Figure 3H-J, Figure S6P and S6Q). We concluded that B.p. induces Th1 differentiation and, together with anti-CTLA-4, activation of Th1 T cells. Interestingly, a recently defined consortium of eleven bacteria was found to induce IFN- $\gamma$ production preferentially in $\mathrm{CD}^{+} \mathrm{T}$ cells and promote anti-tumor immunity in the absence of immunotherapy (Tanoue et al., 2019). In contrast, B.p.-induced IFN- $\gamma$ production in both CD4 ${ }^{+}$ and $\mathrm{CD}^{+} \mathrm{T}$ cells (Figure S6R), and ICB treatment was required for tumoricidal function.

We were intrigued by the ability of B.p. to induce Th1 transcriptional differentiation during

167 homeostasis versus activation of effector function following ICB treatment. Gastrointestinal

168 inflammation is a common immune-related adverse effect of anti-CTLA-4 treatment (Hodi et al., 2010) and we reasoned that this may be due to alterations in gut barrier integrity. Indeed, animals treated with anti-CTLA-4 had increased systemic serum anti-commensal antibodies, particularly Th1-associated IgG2b (Germann et al., 1995), and reduced small intestinal

172 transepithelial electrical resistance compared to controls (Figure S7A and S7B). Although anti-

173 CTLA-4 treatment caused impairment of intestinal barrier integrity it did not induce local or systemic inflammation (Figure S7C and S7D). Since bacteria did not accumulate in the 
175 (heterotopic) tumors and anti-CTLA-4 reduced the integrity of the gut barrier, we hypothesized that increased systemic translocation of metabolites may be responsible for the systemic effect of B.p. during ICB therapy. To address this, we collected serum from tumor-bearing GF, B.p. or C.sp. monocolonized mice treated with anti-CTLA-4 (see Figure 2A) and transferred it 179 concomitantly with anti-CTLA-4 into GF MC38 tumor-bearing mice. Remarkably, serum from

180 CTLA-4-treated B.p. monocolonized mice, but not from GF or C.sp. monocolonized mice, was sufficient to reduce tumor growth and elicit strong anti-tumor immunity in the tumor and spleen of GF mice (Figure 3K-N, Figure S8A-S8F). In sum, these data show that soluble factors derived from or induced by B.p. were responsible for the observed ICB-promoting effects.

Molecular mechanism underlying Th1 immune cell differentiation

In order to identify putative metabolites that might be responsible for the anti-tumor effects of the transferred serum, we determined the metabolomic profile of the transferred serum samples and identified metabolites that were increased in the serum of mice monocolonized with B.p. compared to C.sp. or GF mice. Untargeted metabolomics analysis revealed increased levels of several metabolites in sera from B.p. compared to C.sp. monocolonized and GF mice (Figure 4A, Figure S9A and S9B). Notably, the purine metabolite inosine was the only metabolite that was significantly more abundant ( 8 to 9 -fold) in sera from B.p. monocolonized mice compared to sera from C.sp. monocolonized or GF mice (Figure 4B). Of note, xanthine and hypoxanthine,

193 degradation products of inosine, were also elevated in the sera of B.p. monocolonized mice

194 (Table S2). Analysis of bacterial culture supernatant revealed that B.p. produced approximately ten-fold higher amounts of inosine than C.sp. cultured under the same culture conditions, revealing that inosine is a bacterial metabolite produced by B.p. Of note, L.j. produced higher amounts of hypoxanthine, a potent ligand binding to the same receptor as inosine. (Figure S9C).

198 The identity of inosine was confirmed by fragmentation analysis (Figure S9D). To determine physiological inosine levels in vivo we next measured inosine concentrations in duodenal, 
jejunal and cecal contents of B.p. monocolonized mice. Inosine concentrations were highest in

201 the duodenum and gradually decreased along the gastrointestinal tract (duodenum $66.13 \pm 14.23$

$202 \mu \mathrm{M}>$ jejunum $29.26 \pm 9.38 \mu \mathrm{M}>$ cecum $0.5 \pm 0.05 \mu \mathrm{M}$; Figure $\mathrm{S} 9 \mathrm{E}$ ). We also quantified inosine

concentrations in the serum of B.p. $(26.16 \pm 3.32 \mu \mathrm{M})$ and C.sp. $(3.26 \pm 1.01 \mu \mathrm{M})$

monocolonized mice (Figure S9E), verifying our previous results. Furthermore, inosine levels in the serum of SPF mice $(4.08 \pm 1.12 \mu \mathrm{M})$ increased significantly following anti-CTLA-4 treatment $(11.65 \pm 2.09 \mu \mathrm{M})$ and this was greatly diminished in antibiotic-treated SPF mice $(2.03 \pm 0.86 \mu \mathrm{M})($ Figure $\mathrm{S} 9 \mathrm{~F})$. These data indicated that bacterial production in the upper gastrointestinal tract is likely to be the predominant source of systemic inosine in B.p. monocolonized mice.

210 We next investigated whether inosine could directly enhance anti-tumor Th1 cell

211 differentiation. To test this, we co-cultured activated OVA323-339 peptide-pulsed bone marrow

212 derived dendritic cells (BMDCs) with naïve OVA $323-339$-specific OT-II CD4 ${ }^{+} \mathrm{T}$ cells in the

213 presence or absence of inosine. Intriguingly, inosine led to context-dependent induction or

214 inhibition of $\mathrm{CD}^{+} \mathrm{T}$ cell differentiation. Specifically, in the presence of exogenous IFN- $\gamma$,

215 inosine strongly boosted Th1 differentiation of naïve T cells (Figure 4C) whereas in the absence

216 of IFN- $\gamma$, inosine reduced Th1 induction (Figure 4D and Figure S10A). We then dissected the

217 molecular mechanism through which inosine enhanced Th1 differentiation and found that

218 addition of ZM241385, a pharmacological inhibitor of adenosine $A_{2 A}$ receptor $\left(A_{2 A} R\right)$

219 signaling, completely abrogated the effect of inosine (Figure 4C). Moreover, addition of cell

220 permeable cyclic AMP (db-cAMP), a signaling molecule downstream of A2AR, restored Th1

221 differentiation and bypassed the need for inosine. In addition, inhibition of protein kinase A

222 (PKA), a downstream effector molecule of cAMP, similarly negated inosine-driven Th1

223 differentiation (Figure 4C). Lastly, the inosine-A $\mathrm{A}_{2} \mathrm{R}$-cAMP-PKA signaling cascade led to

224 phosphorylation of the transcription factor cAMP response element-binding protein (CREB) 
(Figure 4E), a known transcriptional enhancer of key Th1 differentiation factors such as IL-12 receptor and IFN- $\gamma$ (Samten et al., 2005; Samten et al., 2008; Yao et al., 2013). Indeed, inosinedependent upregulation of IL12R $\beta 2$ was also observed (Figure S10B). The effect of inosine was $\mathrm{T}$ cell-intrinsic and was not mediated indirectly through DCs because the addition of inosine to naïve $\mathrm{T}$ cells that had been activated with anti-CD3/anti-CD28-coated beads also enhanced Th1 differentiation, even in the absence of IFN- $\gamma$ (Figure S10C). Furthermore, induction of Th1 differentiation and phosphorylation of CREB was absent when $\mathrm{A} 2{ }_{\mathrm{A}} \mathrm{R}$ deficient T cells were stimulated with inosine (Figure S10D and S10E). In contrast, bypassing the need for $\mathrm{A} 2{ }_{\mathrm{A}} \mathrm{R}$ signaling by using db-cAMP increased Th1 differentiation and phosphorylation of CREB in $\mathrm{A} 2{ }_{\mathrm{A}} \mathrm{R}$-deficient $\mathrm{T}$ cells, confirming that the Th1 promoting effect of inosine is depended on $\mathrm{A} 2{ }_{\mathrm{A}} \mathrm{R}$ signaling (Figure S10D and S10E). In addition, since pCREB is known to bind to key Th1 target genes, we also confirmed that inosine stimulation led to a sustained upregulation of Il12rb2 and Ifng gene transcription in CD4 T cells (Figure S10F and S10G). Importantly, inosine dose response experiments revealed that the physiological concentrations of inosine observed in sera of B.p. but not C.sp. monocolonized mice were

240 sufficient to induce Th1 activation (Figure $\mathrm{S} 10 \mathrm{H}$ ). Since adenosine also binds to the A2 $\mathrm{A}$ we 241 also measured adenosine levels and found extremely low levels in intestinal contents and, 242 importantly, no differences in serum levels between B.p. and C.sp. monocolonized mice 243 (Figure S10I), indicating that adenosine could not be mediating the ICB-promoting effects of 244 B.p. Furthermore, adenosine dose-response experiments revealed that the levels of adenosine 245 in the serum were insufficient to promote Th1 activation and effector function (Figure S10J).

246 We next wondered if inosine could also directly affect tumor cell survival or susceptibility to

247 T cell-mediated killing. Direct exposure of MC38 tumor cells to inosine in vitro did not exert 248 any effects on tumor cell viability (Figure S11A). In addition, pretreatment of MC38 tumor 249 cells prior to co-culture with activated tumor-specific T cells did not promote or inhibit T cell- 
mediated killing of tumor cells (Figure S11B). These data indicate that the anti-tumor effect of inosine is mediated through $\mathrm{T}$ cells.

252 Combined, these data suggest that the effect of inosine on $\mathrm{T}$ cells required sufficient costimulation (likely by DCs), IL-12 receptor engagement for Th1 differentiation and IFN- $\gamma$ production for efficient anti-tumor immunity. Classical dendritic cells (cDCs) were found to be the primary source of IL-12 compared to macrophages (Figure S12A and S12B). Thus, we evaluated the impact of cDCs during cancer and ICB-bacteria co-therapy. To do so, bone marrow (BM) cells from cDC-DTR mice were transferred into lethally $\gamma$-irradiated recipient SPF mice to allow for inducible, conditional depletion of cDCs. Following BM reconstitution, mice were treated with antibiotics and then gavaged with a mixture of the three previously identified ICB-promoting bacteria, B.p., L.j., and O.sp. Ten weeks after $\gamma$-irradiation, mice were subcutaneously injected with MC38 CRC cells and when palpable tumors were established, cDCs were depleted by injection of diptheria toxin followed one day later by antiCTLA-4 treatment (Figure 4F). Depletion of cDCs led to a significant reduction in intratumoral $\mathrm{CD}^{+}$and $\mathrm{CD}^{+} \mathrm{T}$ cell frequencies and IFN- $\gamma$ production (Figure $4 \mathrm{G}-\mathrm{J}$ ), which resulted in larger tumors (Figure 4K). Similarly, IFN- $\gamma$ production and proliferation of splenic $\mathrm{CD}^{+}$and $\mathrm{CD}^{+} \mathrm{T}$ cells were also markedly reduced in cDC-depleted animals (Figure S12C-S12F). Therefore, depletion of cDC strongly reduced the efficacy of bacteria-elicited ICB to reduce established tumors, which indicates the requirement for continuous antigen presentation, IL-12 production and $\mathrm{T}$ cell co-stimulation by $\mathrm{cDCs}$ for efficient ICB therapy.

Inosine promotes Th1 immunity and tumoricidal effects in vivo

271 To confirm whether the inosine-mediated Th1 promoting effect in vitro also applied to in vivo

272 conditions, GF mice were immunized with ovalbumin in combination with $\mathrm{CpG}$ as a co-

273 stimulus. One day later mice received inosine or vehicle only intraperitoneally. Inosine 274 increased the proportions of T-bet ${ }^{+}, \mathrm{IFN}-\gamma^{+} \mathrm{CD} 8^{+}$and $\mathrm{CD}^{+} \mathrm{T}$ cells in the MLN (Figure 5A- 
275 C), validating our in vitro results. In order to assess whether the effect of inosine also promoted

276 anti-tumor immunity, we challenged GF mice with MC38 tumor cells. Upon palpable tumors,

277 inosine or PBS was given orally or systemically in combination with anti-CTLA-4 treatment

278 and $\mathrm{CpG}$ as indicated (Figure 5D). Compared to PBS, inosine led to a reduction of tumor weight

279 and increased anti-tumor immunity irrespective of oral or systemic application routes when

280 given together with anti-CTLA-4 and CpG (Figure 5E-G and Figure S12G and S12H). In

281 contrast, in the absence of $\mathrm{CpG}$ as a co-stimulus, inosine increased tumor weight and reduced

282 anti-tumor immunity (Figure 5 E-G and Figure S12G and S12H). These results validate our

283 previous in vitro findings demonstrating that the effect of inosine is context dependent based

284 on the amount of co-stimulation present.

285 We then confirmed that inosine-induced anti-tumor immunity was dependent on $\mathrm{A} 2{ }_{\mathrm{A}} \mathrm{R}$ signaling in vivo. Germ-free Rag1-deficient animals were challenged with MC38 tumor cells and simultaneously received WT or A2AR-deficient T cells. Seven days later, inosine was given orally in combination with anti-CTLA-4 and CpG (Figure 5H). Inosine enhanced anti-CTLA4/CpG-mediated anti-tumor immunity in animals that received WT but not $\mathrm{A} 2{ }_{\mathrm{A}} \mathrm{R}$-deficient $\mathrm{T}$

290 cells (Figure 5I-L). Specifically, in the presence of inosine, WT but not A2 AR-deficient T cells displayed increased IFN- $\gamma$ production within tumors and subsequently only GF mice receiving WT T cells showed reduced tumor weights (Figure 5I-L). Moreover, B.p. promoted anti-

293 CTLA-4 efficacy in tumor bearing Ragl-deficient mice transfused with WT but not A2 ${ }_{\mathrm{A}} \mathrm{R}$ -

294 deficient T cells (Figure 5M-P). This indicated that the ICB-promoting effect of B.p. was 295 dependent on $\mathrm{A} 2{ }_{\mathrm{A}} \mathrm{R}$ signaling and demonstrated a dependency on $\mathrm{A} 2{ }_{\mathrm{A}} \mathrm{R}$ signaling specifically in T cells for the anti-tumor effect of B.p.-inosine-ICB co-therapy.

297 Taken together, inosine-A2 $\mathrm{A}$ signaling drives or inhibits anti-tumor immunity in vivo, 298 depending on the amount of co-stimulation present. 


\section{Differential effect of ICB-promoting bacteria on CRC subtypes}

300 Lastly, we examined the effect of the identified ICB-promoting bacteria in two distinct models

301 of CRC that mimic different subtypes of human CRC. First, we tested the ICB-promoting effect

302 of B.p., L.j., and O.sp. in Apc ${ }^{2 l o x 14 /+} ; K_{r a s}^{L S L-G 12 D /+} ;$ Fabpl-Cre (Haigis et al., 2008) SPF mice,

303 which have conditional Apc deficiency and activation of Kras specifically in colonocytes. In

304 this model of CRC, anti-CTLA-4 treatment alone did not improve survival compared to

305 isotype-treated animals (Figure 6A and B). To test if the addition of ICB-promoting bacteria

306 could switch a non-responsive to a responsive effect in this model, SPF mice were treated with

307 a mixture of broad-spectrum antibiotics for 7 days to overcome colonization resistance(Lee et

308 al., 2013), followed by bacterial transfer and treatment with anti-CTLA-4. Although the ICB-

309 promoting bacteria colonized the intestine, this combined approach did not enhance survival

310 (Figure 6C and D), revealing a limitation of bacterial co-therapy in this model. Next, we

311 examined the effect of ICB-promoting bacteria in SPF Msh2 $2^{\text {LoxP/LoxP }}$ Villin-Cre (Kucherlapati

312 et al., 2010) animals that have conditional inactivation of $M s h 2$ in intestinal epithelial cells. In

313 this model, anti-CTLA-4 treatment alone (without the addition of ICB-promoting bacteria) led

314 to reduced tumor weight and cancer stem cells and increased T cell activation and immune cell

315 infiltration in the tumor (Figure 6E-G, Figure S13A, S13C and S13E). Remarkably, co-

316 treatment with ICB-promoting bacteria boosted the effect of anti-CTLA-4, leading to a further

317 marked reduction of tumor weight and cancer stem cell numbers together with drastically

318 enhanced $\mathrm{T}$ cell activation and immune cell infiltration in the tumor compared to control

319 bacteria (Figure 6H-J, Figure S13B, S13D and S13F). In support of a critical role for inosine-

320 dependent upregulation of IL12R $\beta 2$ on T cells and cDC IL-12 production and function, anti-

321 IL-12p75 treatment almost completely abrogated the effect of ICB-promoting, anti-CTLA-4

322 co-therapy in $M s h 2^{\text {LoxP/LoxP } V i l l i n-C r e ~ t u m o r s, ~ w h i c h ~ c o r r o b o r a t e s ~ s i m i l a r ~ f i n d i n g s ~ u p o n ~}$

323 simultaneous depletion of IL-12 and IL-23, using anti-IL-12p40 treatment (Routy et al., 2018; 
324 Vetizou et al., 2015). Finally, since oxaliplatin-anti-PD-L1 co-treatment is a more commonly used therapy in the clinics, we confirmed that ICB-promoting bacteria also promoted the

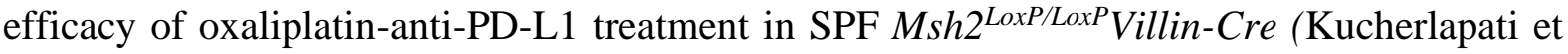
al., 2010) animals (Figure S14).

In summary, our results reveal a novel bacterial-inosine-immune pathway that boosts a cDCdependent Th1 T cell circuit to greatly enhance the effect of ICB therapies in CRC (Figure S15).

\section{$\underline{\text { Discussion }}$}

332 ICB therapy has yielded rather disappointing results in CRC, with an objective response only 333 in $40 \%$ of patients with the mismatch repair deficient (MMRD) sub-type of CRC, which amounts to only $4 \%$ of all CRC (Le et al., 2015). We have now identified a novel microbialmetabolite-immune circuit that enhances ICB therapy in two mouse models of CRC. These data indicate that modification of the microbiota may provide a promising adjuvant therapy to ICB in CRC. Of note, compared to anti-PD-L1, anti-CTLA-4 induced stronger anti-tumor

338 effects in the AOM/DSS and heterotopic tumor models when both antibodies were administered at the same dose. At this point it is difficult to know if this is due to differences in the biological effects of blocking CTLA-4 versus PD-L1 in these models, but it should be noted that other experimental studies routinely use anti-PD-1 mAb at much higher does than anti-CTLA4 mAb (Routy et al., 2018).

343 By isolating tumor-associated bacteria we have identified several bacterial species that were found to be associated exclusively with tumors following treatment with ICB, with three of these bacteria able to significantly enhance the efficacy of ICB therapy in CRC. This suggests that the isolation of bacterial species from intestinal tumor biopsies rather than from feces may

347 be a better approach in a clinical setting for defining ICB-promoting bacteria in CRC. Although 348 isolated from mice, all three ICB-promoting bacteria are also found in humans, indicating their 
potential for translation (Dewhirst et al., 2001; Pridmore et al., 2008; Turroni et al., 2009).

Although Bifidobacterium species, such as B. breve and B. longum, have previously been

been reported to provide protection from anti-CTLA-4-induced enterocolitis with no effect on tumor growth(Wang et al., 2018). Bifidobacterium pseudolongum species are widely distributed in the mammalian gut with many different strains displaying genomic diversity and differential metabolic capacities (Lugli et al., 2019), suggesting strain-dependent functions and a need for a precision approach to microbial therapy. Lactobacillus johnsonii has not previously been associated with anti-tumor immunity; in contrast, it has been shown to have antiinflammatory effects (Bereswill et al., 2017). Much less is known about the functions of Olsenella species.

360 Our findings demonstrate a critical role for the bacterial metabolite inosine in setting a baseline

361 Th1 level in local mucosal tissues. Initially, this was surprising because previous reports have demonstrated an inhibitory effect of inosine, and $\mathrm{A}_{2 \mathrm{~A}} \mathrm{R}$ engagement in general, on $\mathrm{Th} 1$ differentiation in vitro and anti-tumor immunity in vivo (Csoka et al., 2008; Hasko et al., 2000;

364 He et al., 2017; Ohta et al., 2006). Indeed, the wealth of data supporting an immunosuppressive role for adenosine and $\mathrm{A} 2 \mathrm{~A} \mathrm{R}$ signaling has led to the development of novel immune checkpoint inhibitor targets, such as mAb targeting CD73, CD39 and CD38, and pharmacological antagonists of $\mathrm{A} 2{ }_{\mathrm{A}} \mathrm{R}$, many of which are currently in clinical trials (reviewed in(Vigano et al., 2019)). However, a small body of literature has demonstrated that inosine can be pro-

369 inflammatory and $\mathrm{A} 2{ }_{\mathrm{A}} \mathrm{R}$ signaling can sustain Th1/anti-tumor immunity in mice (Cekic and 370 Linden, 2014; Lasek et al., 2015; Lioux et al., 2016). Our findings reconcile these contrasting 371 observations by revealing a context-dependent effect of inosine-A2 A receptor signaling based 372 on the amount of co-stimulation. Mechanistically, inosine engages the $\mathrm{A} 2 \mathrm{~A}$ receptor and 373 activates the transcription factor CREB, through cAMP. CREB, together with co-factors and 
the formation of heterodimers with ATF-2 and/or c-Jun, modulates the transcription of key Th1 genes, including Il12rb2 and Ifng (Samten et al., 2008). It is worth noting that in addition to cAMP signaling, inosine (compared to adenosine) has a distinct $\mathrm{A} 2{ }_{\mathrm{A}} \mathrm{R}$-dependent signaling bias, with a 3.3-fold preference for ERK1/2 phosphorylation. In light of our findings, blockade of inosine-A2 $\mathrm{A}$ receptor signaling in cancer immunotherapy could negate a positive effect provided by beneficial microbes. We suggest that $\mathrm{A} 2 \mathrm{~A}$ receptor signaling is likely an integral anti-tumor pathway for bacterial-ICB co-therapies. Indeed, Tanoue et al recently identified a consortium of eleven bacteria that improve ICB therapies (Tanoue et al., 2019), which are not related to the bacteria identified in this work. Remarkably though, two of the most elevated metabolites in the cecum and serum of mice colonized with the consortium of 11 bacteria were inosine monophosphate and hypoxanthine, a substrate and product of inosine respectively, which are both $\mathrm{A} 2 \mathrm{~A}$ receptor agonists like inosine (Welihinda et al., 2016). The identification of this context-dependent effect of inosine-A2A receptor signaling is particularly relevant as inosine is currently used as an intervention in clinical trials in various Th1-associated diseases, including multiple sclerosis, amyotrophic lateral sclerosis and Parkinson's disease (Bettelli et al., 2004; Kustrimovic et al., 2018; Lovett-Racke et al., 2004; Saresella et al., 2013). We identified cDCs and their production of IL-12 as essential components for efficient induction of anti-tumor T cell immunity elicited by ICB therapy in the presence of beneficial bacteria. The critical involvement of cDC and IL-12 has also been recently reported upon antiPD-1 treatment (Garris et al., 2018).

394 Seminal work by Guinney et. al revealed four molecular consensus subtypes of CRC(Guinney et al., 2015), namely MMRD, canonical, metabolic and mesenchymal. In line with the positive results of ICB in MMRD patients in the clinical setting(Le et al., 2015), in our animal model of MMRD (Msh2 $\left.2^{\text {LoxP/LoxP } V i l l i n-C r e ~}\right)$ we indeed observed some efficacy of anti-CTLA-4 single

398 therapy. However, co-therapy with ICB-promoting bacteria strongly enhanced the tumoricidal 
effect of anti-CTLA-4. Thus, bacterial co-therapy may optimize treatment regimens in MMRD

400 CRC patients. Secondly, ICB therapy was efficacious and was associated with $B$.

tumors have been used to model inflammation-associated CRC. AOM/DSS tumors also display characteristics of epithelial to mesenchymal transition (Lin et al., 2015), such as reduced Eincreased TGF- $\beta$ expression (Becker et al., 2004; Mager et al., 2017), which are hallmarks of indicate a benefit of bacterial co-therapy also in this subtype. Canonical and metabolic CRC subtypes are both characterized by inactivation of $A p c$, canonical additionally by Wnt pathway and metabolic by KRAS activation (Guinney et al., 2015). These hallmarks are well represented in the $\mathrm{Apc}^{2 l o x 14 /+} ; \mathrm{Kras}^{L S L-G 12 D /+} ; \mathrm{Fabpl}$-Cre animal model and intriguingly

411 bacterial co-therapy did not improve anti-CTLA-4 treatment. The divergent effect of ICB-

412 promoting bacteria in the Msh2 $2^{\text {LoxP/LoxP }}$ Villin-Cre- compared to the Apc ${ }^{2 l o x 14 /+} ; \mathrm{Kras}^{\text {LSL-G12D/+ }}$;

413 Fabpl-Cre model is intriguing and at this stage we can only speculate about the underlying 414 reason(s). The mutational load and associated number of neoantigens, which is likely higher in $415 M s h 2^{\text {LoxP/LoxP } V i l l i n-C r e ~ t u m o r s, ~ c e r t a i n l y ~ i m p a c t s ~ o n ~ t h e ~ e f f i c a c y ~ o f ~ I C B ~ t h e r a p i e s ~(H a v e l ~ e t ~ a l ., ~}$ 2019). Moreover, anti-CTLA-4 had no effect on its own in the $\mathrm{Apc}^{2 l o x 14 /+}$; $\mathrm{Kras}^{\mathrm{LSL}-\mathrm{Gl} 2 \mathrm{D} /+}$;

417 Fabpl-Cre model and bacteria alone did not impact on heterotopic tumor development. We 418 also showed that B.p. increased the Th1 cell pool and their anti-tumor effect was unleashed 419 followed by effective ICB therapy. Thus, we reason that the discovery of novel checkpoint 420 blockade targets or other therapies that have an effect of their own in the $\mathrm{Apc}^{2 l o x 14 /+} ; \mathrm{Kras}^{L S L-}$ ${ }^{\mathrm{Gl2D/+}}$; Fabpl-Cre model are required to enable efficacious bacterial co-therapy to treat similar subtypes in CRC patients.

423 Together, this work paves the way for new approaches to precision medicine in CRC. 


\section{Acknowledgements}

425 We are grateful to Carolyn Thomson, Aline Ignacio Silvestre da Silva, Madeleine Wyss, 426 Marcela Davoli-Ferreira, Jenine Yee and Mia Koegler for their help in tackling large scale 427 experiments, their technical knowledge and critical feedback. We thank Mike Dicay for 428 performing the Ussing chamber experiments. Metabolomics data were acquired by RAG at the 429 Calgary Metabolomics Research Facility (CMRF), which is supported by the Canada 430 Foundation for Innovation (JELF 34986) and the International Microbiome Centre (IMC). The 431 IMC is supported by the Cumming School of Medicine, University of Calgary, Western 432 Economic Diversification (WED) and Alberta Economic Development and Trade (AEDT), 433 Canada. LFM was supported by the Early Postdoc.Mobility Fellowship from the Swiss 434 National Science Foundation. KB is supported by a Canada Graduate Scholarship from the 435 National Sciences and Engineering Research Council of Canada (NSERC). MBG is supported 436 by a Canadian Institutes of Health Research (CIHR) grant (PJT-391060) and a Canadian 437 Foundation for Innovation (CFI) grant. KDM is supported by a CIHR grant (PJT-420305), a 438 CFI grant and the Cumming School of Medicine. I.A.L. is supported by an Alberta Innovates 439 Translational Health Chair. JS is supported by a CIHR grant and a Terry Fox Research Institute 440 grant.

\section{Authors' contribution}

442 LFM, RB, NC, KB, HR, SP, RAG, IAL, and MG performed experiments and analyzed data.

443 LFM, MBG and KDM wrote the manuscript, and all authors revised the manuscript and 444 approved its final version. JS provided A2AR-deficient mice. LFM and KDM conceived the 445 project. KDM and MBG supervised the project. 
bioRxiv preprint doi: https://doi.org/10.1101/2020.02.26.965533; this version posted February 27, 2020. The copyright holder for this preprint (which was not certified by peer review) is the author/funder, who has granted bioRxiv a license to display the preprint in perpetuity. It is made available under aCC-BY-NC-ND 4.0 International license.

\section{$446 \quad$ Declaration of Interests}

447 JS is a permanent member of the Scientific Advisory Board of Surface Oncology and owns

448 stocks of Surface Oncology. LFM and KDM have filed a patent application for the use of 449 probiotics and metabolites as checkpoint blockade adjuvants.

450 


\section{Figure legends}

Figure 1
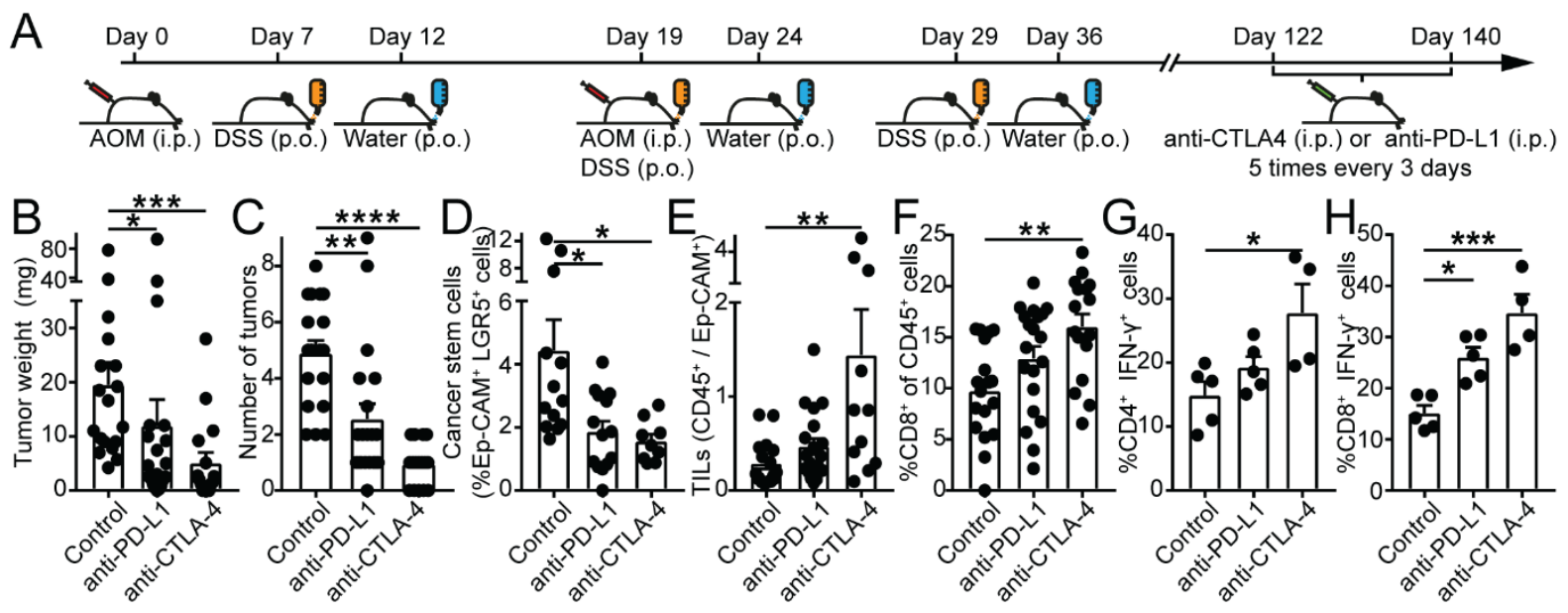

I
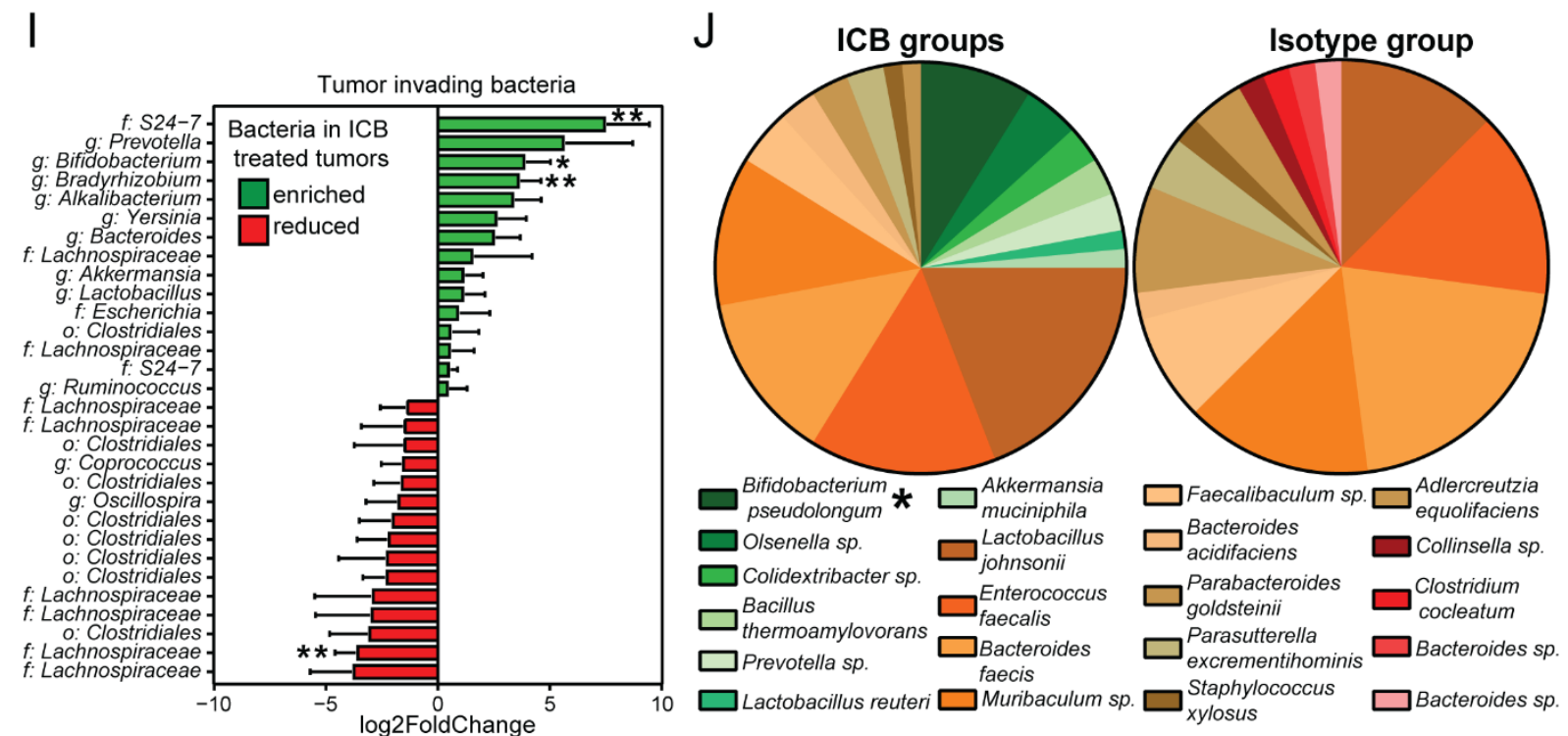

Figure 1: Immune cell and microbial dynamics upon ICB therapy in AOM/DSS tumors. (A) Overview of the experimental setup of AOM/DSS-induced CRC and ICB treatment. (B) Tumor weight, (C) number of tumors, (D) Ep-CAM ${ }^{+}$, LGR5 ${ }^{+}$cancer stem cells and (E) tumorinfiltrating leukocytes (TILs) 140 days post induction in animals treated with isotype-, antiPD-L1 or anti-CTLA-4 antibodies. (F) $\mathrm{CD}^{+} \mathrm{T}$ cell frequencies in the tumor draining lymph node at day 140 . Splenic IFN- $\gamma^{+}$production in $(\mathrm{G}) \mathrm{CD}^{+}$or $(\mathrm{H}) \mathrm{CD}^{+} \mathrm{T}$ cells. (I) $16 \mathrm{~S}$ rRNA gene V4 region amplicon sequencing to identify bacteria in tumor tissue. Bacteria enriched or reduced in tumors of anti-PD-L1/anti-CTLA-4 compared to isotype treated animals are shown in green or red, respectively. ( $\mathrm{J})$ Bacteria cultured from homogenized tumors under anaerobe conditions from anti-PD-L1/anti-CTLA-4 (ICB groups) or isotype (Isotype group) treated animals. Bacteria depicted as green or red could only be cultured in ICB groups or Isotype group, respectively. Bacteria depicted as brown were present in both groups. Data are (B-H) mean \pm SEM or $(\mathrm{I})$ mean \pm lfcSE (logfoldchangeStandard Error) and pooled from three individual experiments. (B-F) $n=16-20$ mice/group, (g and h) $n=4-5$ mice/group. * $P<0.05$; **, $P<0.01$; ***, $P<0.001$; ****, $P<0.0001$. 
A
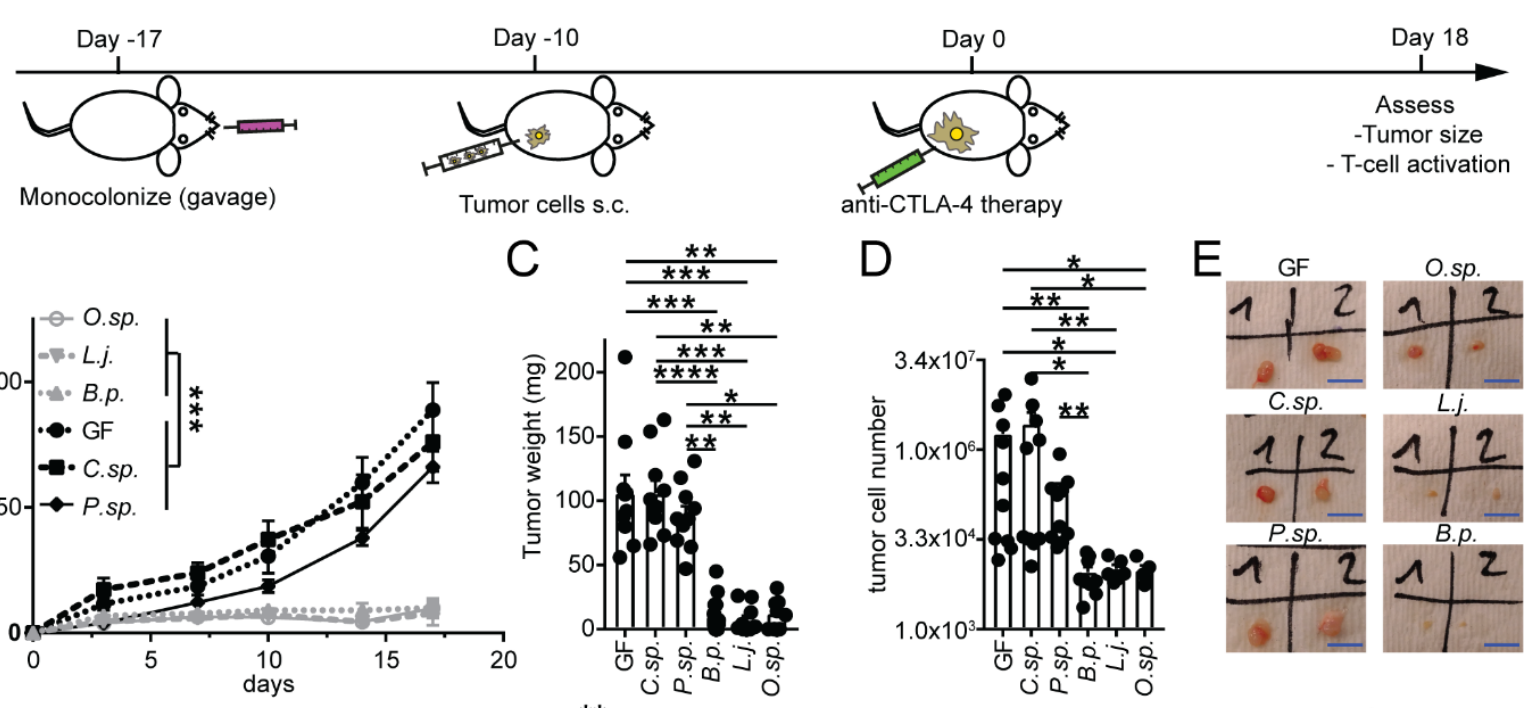

B

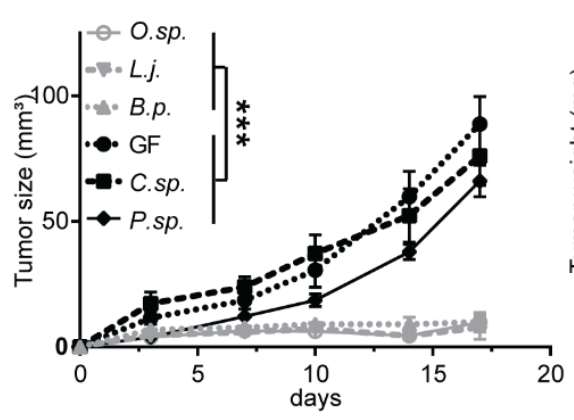

C

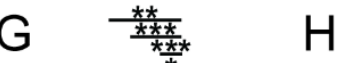

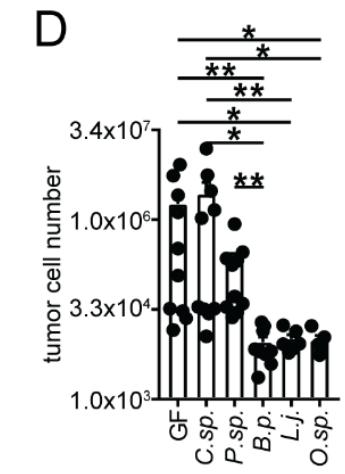

$\mathrm{H} \quad$ GF

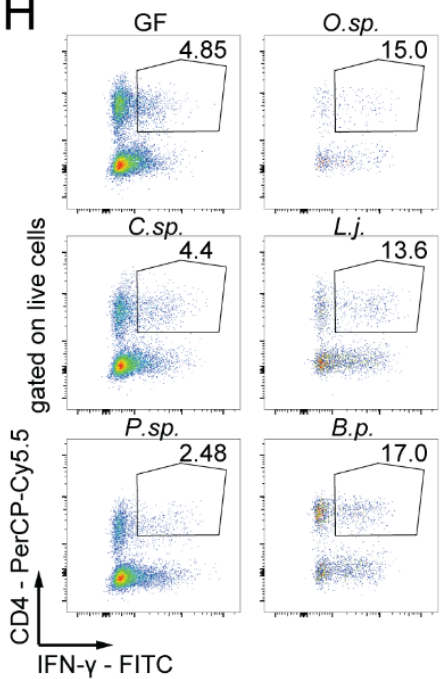

$\mathrm{E}$

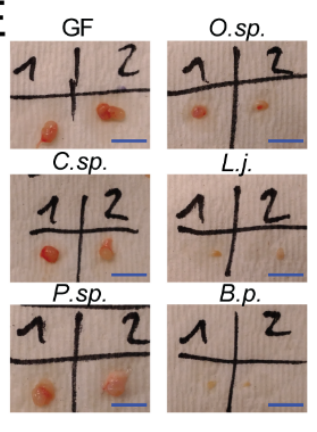

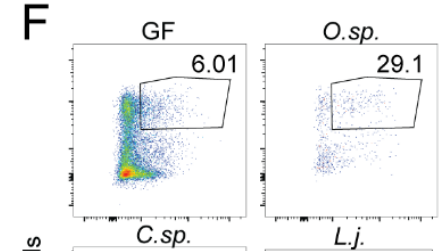
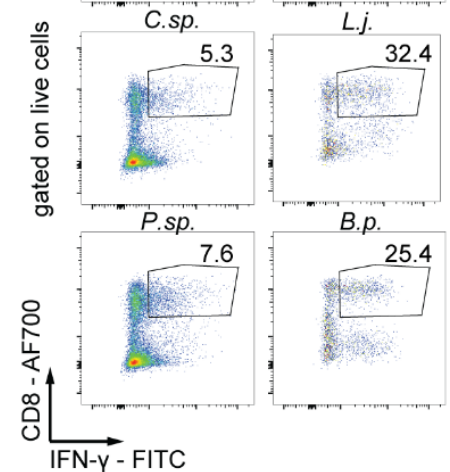

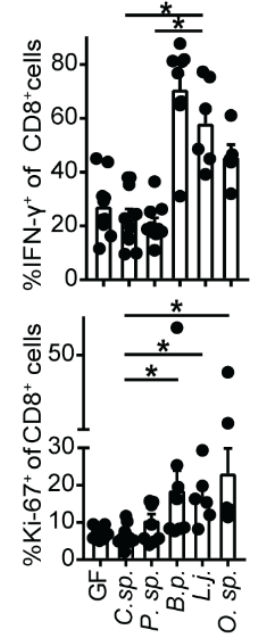

Figure 2: Individual bacterial species boost ICB therapy. (A) Schematic of the experimental setup to assess the effect of individual bacteria on anti-CTLA-4 therapy efficacy. (B) Tumor growth, (C) tumor weight, (D) live tumor cells and (E) representative pictures of tumors are shown at day 18 . Scale bars: $1 \mathrm{~cm}$. (F) representative plots and (G) quantification of IFN- $\gamma^{+}$and $\mathrm{Ki}-67^{+}, \mathrm{CD}^{+} \mathrm{T}$ cells at day 18 in the tumor tissue. (H and I) same as (F and $\left.\mathrm{G}\right)$, but for $\mathrm{CD}^{+}$ $\mathrm{T}$ cells. Data are mean \pm SEM and pooled from three individual experiments $(n=8-15$ mice/group). *, $P<0.05 ; * *, P<0.01 ; * * *, P<0.001 ; * * * *, P<0.0001$. 

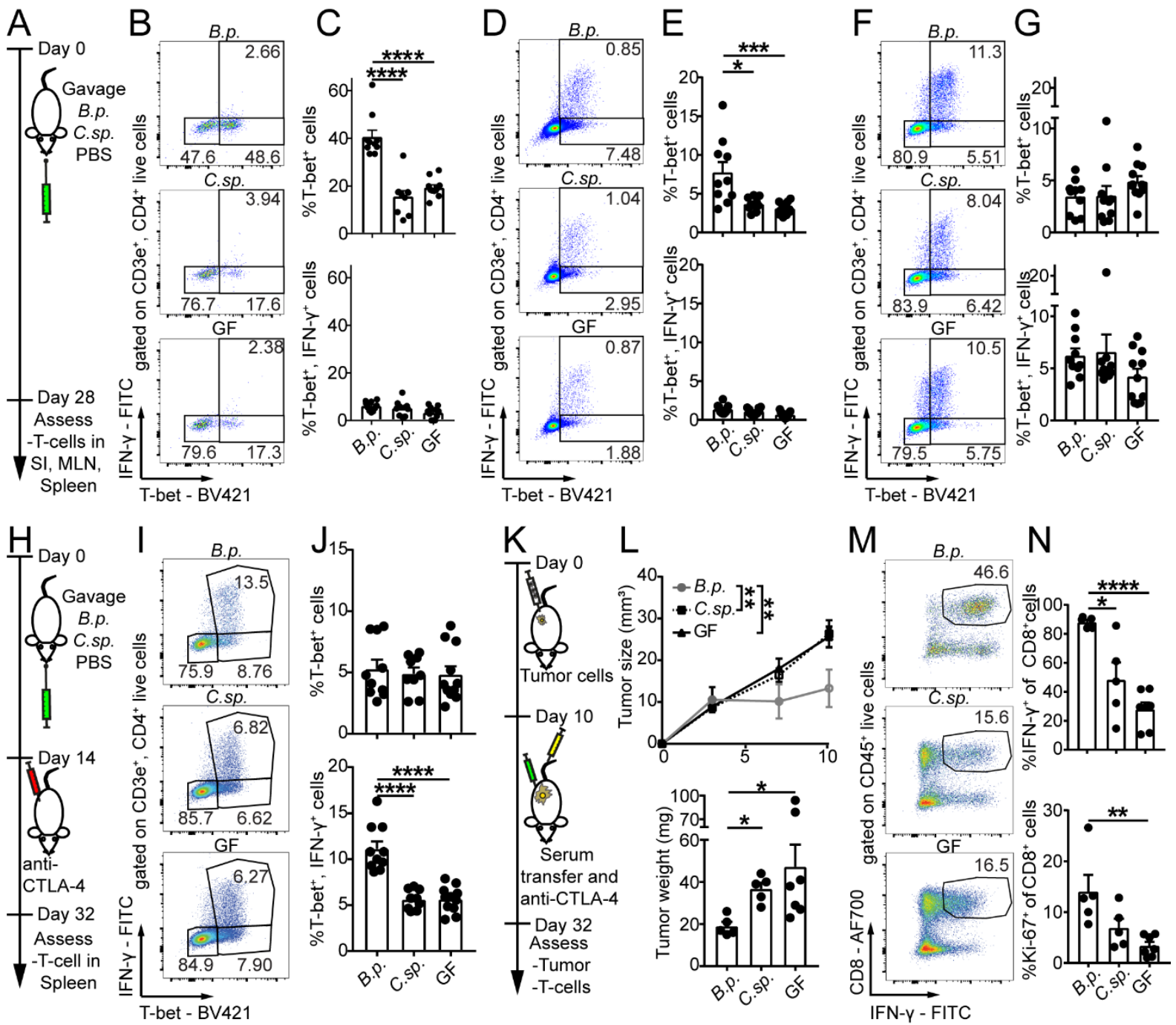

Figure 3: Effect of B.p., anti-CTLA-4 and B.p. conditioned serum on $T$ cell differentiation and activation. (A, $\mathrm{H}$ and $\mathrm{K}$ ) Schematic of the experimental setups. (B) representative plots and (C) quantification of T-bet ${ }^{+}$and T-bet ${ }^{+}, \mathrm{IFN}_{-} \gamma^{+}$events of $\mathrm{CD}^{+}, \mathrm{CD}^{+}$cells in the small intestine (SI) in the presence of indicated bacteria at day 28. (D and E) same as (B and C), but in the mesenteric lymph node (MLN). (F and G) same as (B and C,) but in the spleen. (I) representative plots and (J) quantification of T-bet ${ }^{+}$and T-bet ${ }^{+}, \mathrm{IFN}^{+} \gamma^{+}$events of $\mathrm{CD} 3^{+}, \mathrm{CD} 4^{+}$

$488 \mathrm{~T}$ cells in the spleen in the presence of indicated bacteria and anti-CTLA-4 treatment at day 32.

489 (L) Tumor growth and weight are shown 32 days after MC38 tumor challenge and subsequent 490 serum transfer as well as anti-CTLA-4 treatment. (m) representative plots and (n) quantification 491 of intratumoral IFN $-\gamma^{+}$or Ki- $67^{+} \mathrm{CD}^{+} \mathrm{T}$ cells. Data are mean \pm SEM and pooled from two individual experiments (A-J) $n=10-11$ mice/group, (K-N). $n=5-7$ mice/group. ${ }^{*}, P<0.05$; **, $P<0.01$; ***, $P<0.001$; ****, $P<0.0001$. 

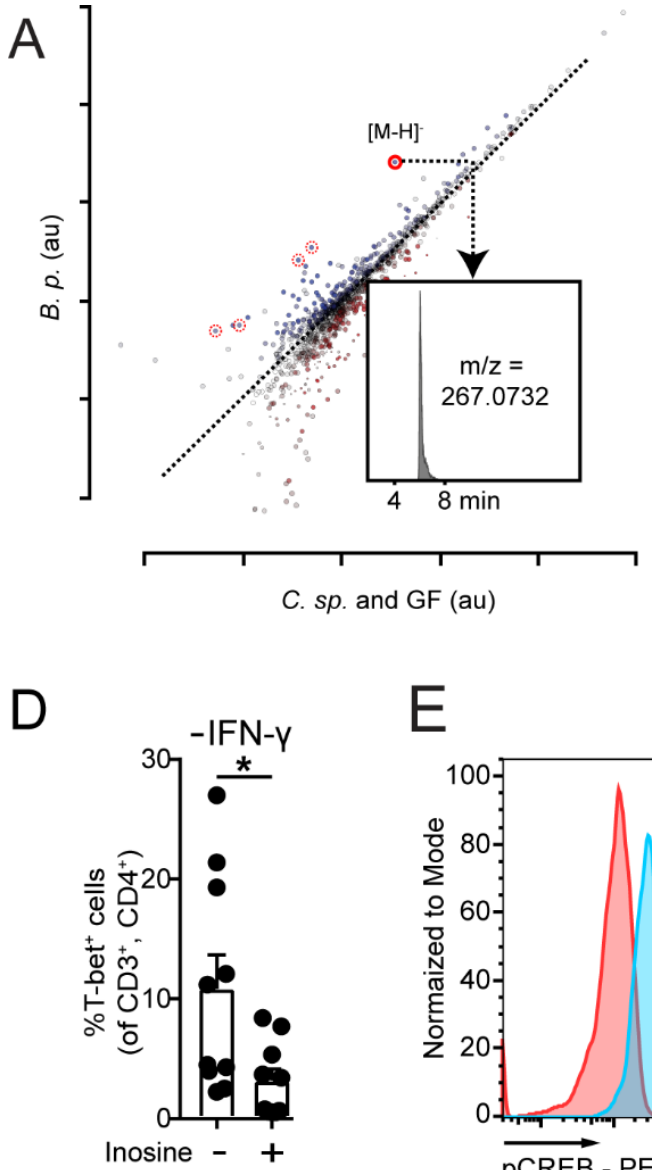
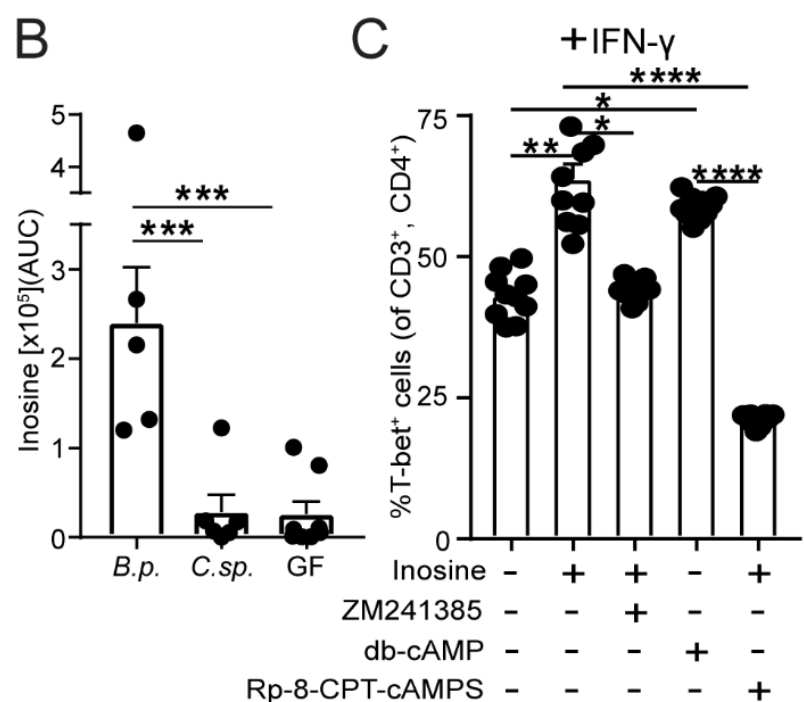
Rp-8-CPT-CAMPS -
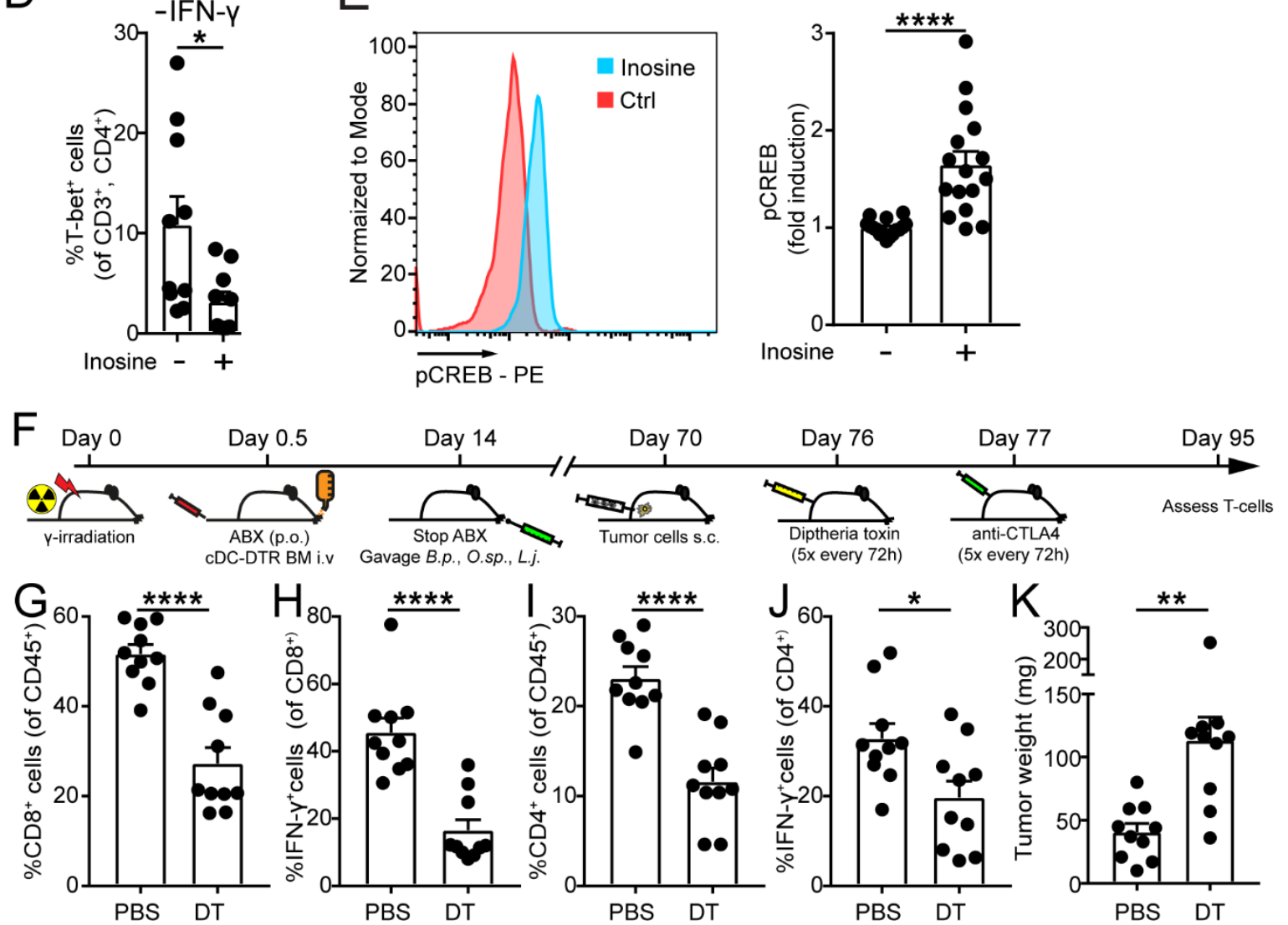

Figure 4: Effect of inosine on $T$ cell differentiation and dependency on classical dendritic cells of ICB therapy efficacy. (A) Scatter plot of untargeted metabolomics data in the serum of anti-CTLA-4 treated, tumor-bearing B.p. monocolonized compared to C.sp. monocolonized and GF mice. Red circles or dotted red circles depict inosine or inosine fragments/adducts, respectively. Inset shows an extracted ion chromatogram of inosine (B) Intensity of inosine (AUC: area under the curve) in sera shown in panel (A) of this figure. (C) Naïve CD4 ${ }^{+} \mathrm{T}$ cells were co-cultured with bone marrow derived dendritic cells and IFN- $\gamma$. Quantification of T-bet ${ }^{+}$, $\mathrm{CD}^{+}, \mathrm{CD}^{+} \mathrm{T}$ cells 48 hours after co-culture in the presence or absence of inosine, $\mathrm{A}_{2 \mathrm{~A}}$ receptor inhibitor (ZM241385), cell permeable cAMP (db-cAMP) and protein kinase A inhibitor (RP-8-CPT-cAMPS). (D) Same as (C) without IFN- $\gamma$. (E) Representative plot and quantification (left and right panel) of phospho-CREB (Ser133) levels in naïve CD4 ${ }^{+} \mathrm{T}$ cells 
508 cultured with anti-CD3/anti-CD28 coated beads for 1 hour in the presence or absence of 509 inosine. (F) Schematic overview of experimental setup to deplete classical dendritic cells 510 during MC38 tumor challenge and anti-CTLA-4 treatment. Intratumoral (G) frequency of $511 \mathrm{CD}^{+} \mathrm{T}$ cells $(\mathrm{H}) \mathrm{IFN}-\gamma^{+} \mathrm{CD} 8^{+} \mathrm{T}$ cells. (I and $\left.\mathrm{J}\right)$ same as $(\mathrm{G}$ and $\mathrm{H})$, but $\mathrm{CD} 4^{+} \mathrm{T}$ cells and $(\mathrm{K})$ 512 tumor weight at day 95. Data are mean \pm SEM and pooled from two individual experiments 513 (A-B) $n=5-8$ samples/group (C-E) 10-15 biological replicates/group (F-K) 10 mice/group. *, $514 \quad P<0.05 ; * *, P<0.01 ; * * *, P<0.001 ; * * * *, P<0.0001$.

515 See also Figures: S9, S10, S11 and S12 
Figure 5
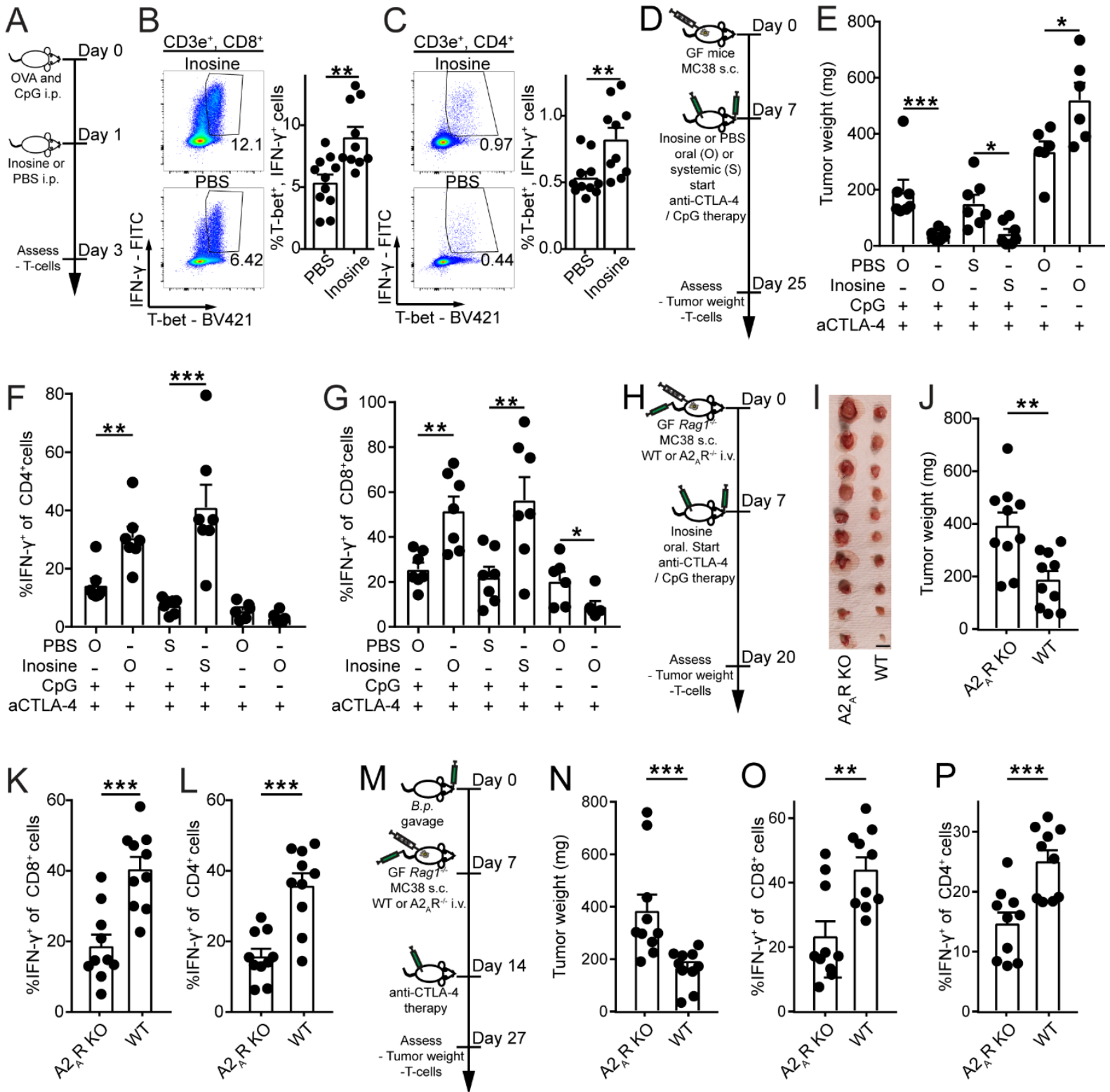

519 Figure 5: Inosine promotes Th1 activation and anti-tumor immunity. (A) Schematic overview of experimental setup to assess the effect of inosine on Th1 activation in vivo. (B and C) Representative dot plots and quantification (left and right panel respectively) of T-bet ${ }^{+}$, IFN$\gamma^{+}, \mathrm{CD}^{+}$(B) $\mathrm{CD}^{+}$or (C) $\mathrm{CD}^{+} \mathrm{T}$ cells in the MLN. (D) Schematic overview of experimental setup to assess the effect of inosine on anti-tumor immunity. Upon palpable tumors, mice were treated with $100 \mu \mathrm{g}$ anti-CTLA-4 i.p. (5 times every 72 hours) and in some groups $20 \mu \mathrm{g}$ CpG i.p. (5 times every 72 hours). In addition, inosine $(300 \mathrm{mg} / \mathrm{KG} / \mathrm{BW})$ or PBS was given daily orally (O) through gavage or systemically (S) through i.p. injection. (E) Tumor weight and quantification of IFN- $\gamma^{+}$cells amongst $(\mathrm{F}) \mathrm{CD}^{+}$or $(\mathrm{G}) \mathrm{CD}^{+}$cells are shown. (H) Schematic overview to assess the requirement of $\mathrm{A} 2{ }_{\mathrm{A}} \mathrm{R}$ signaling for inosine-induced anti-tumor immunity. $1 \times 10^{6} \mathrm{MC} 38$ cells (s.c.) and WT or $\mathrm{A} 2{ }_{\mathrm{A}} \mathrm{R}$-deficient $1 \times 10^{7} \mathrm{~T}$ cells (i.v. $6 \times 10^{6} \mathrm{CD} 4^{+}$ and $4 \times 10^{6} \mathrm{CD}^{+}$cells) were injected. Upon palpable tumors, mice were treated with $100 \mu \mathrm{g}$

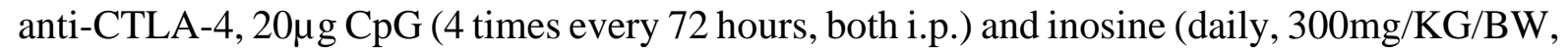
through gavage). (I) Pictures of tumors are shown at day 20. scale bars: $1 \mathrm{~cm}$. (J) Tumor weight 
533 and quantification of IFN $-\gamma^{+}$in $(\mathrm{K}) \mathrm{CD}^{+}$or $(\mathrm{L}) \mathrm{CD}^{+}$cells in the tumor are shown. (M) 534 Schematic overview to assess the requirement of $\mathrm{A} 2 \mathrm{~A}_{\mathrm{A}} \mathrm{R}$ signaling for B.p.-induced anti-tumor 535 immunity. Mice were gavaged with B.p. and seven days later $1 \times 10^{6} \mathrm{MC} 38$ cells (s.c.) and WT 536 or $\mathrm{A} 2 \mathrm{AR}$-deficient $1 \times 10^{7} \mathrm{~T}$ cells (i.v. $6 \times 10^{6} \mathrm{CD}^{+}$and $4 \times 10^{6} \mathrm{CD} 8^{+}$cells) were injected. Upon 537 palpable tumors, mice were treated with $100 \mu \mathrm{g}$ anti-CTLA-4 (4 times every 72 hours). (N) 538 Tumor weight and quantification of $\mathrm{IFN}-\gamma^{+}$in $(\mathrm{O}) \mathrm{CD}^{+}$or $(\mathrm{P}) \mathrm{CD}^{+}$cells in the tumor are 539 shown. Data are mean \pm SEM and pooled from two individual experiments (A-P) 10-11 540 mice/group. *, $P<0.05 ; * *, P<0.01 ; * * *, P<0.001$.

541 See also Figures S12 
Figure 6
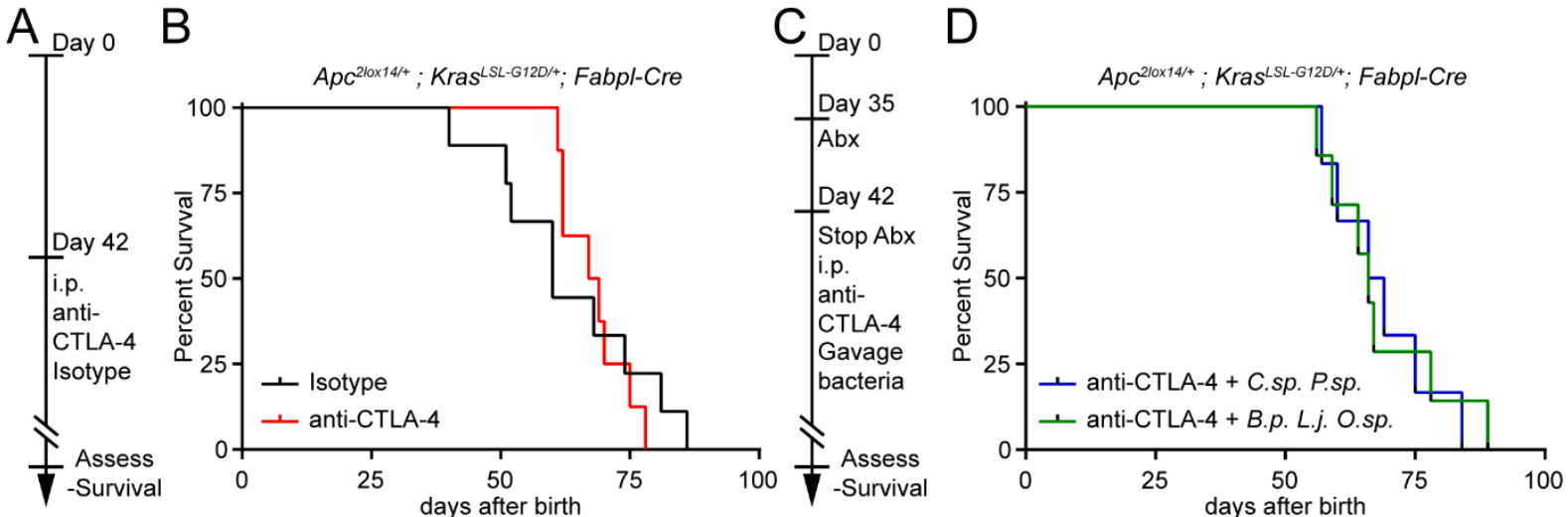

\section{$E$}
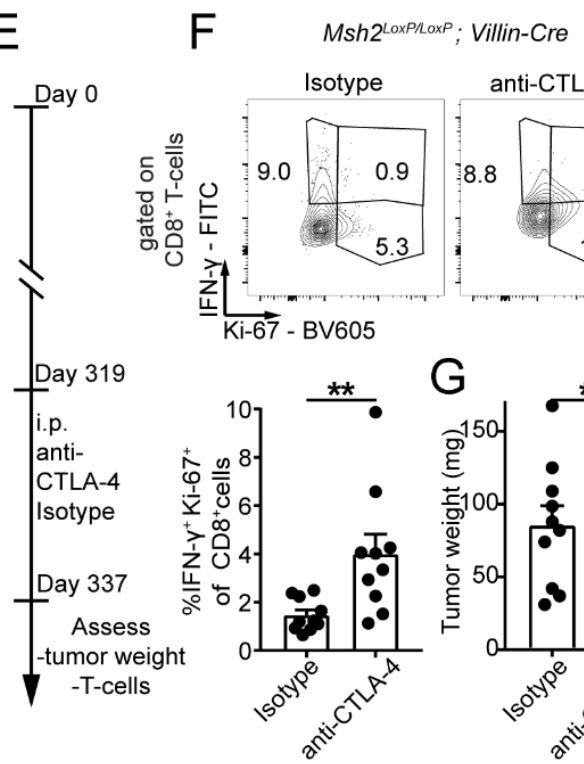

anti-CTLA-4

$\mathrm{H} \quad \mathrm{I}$
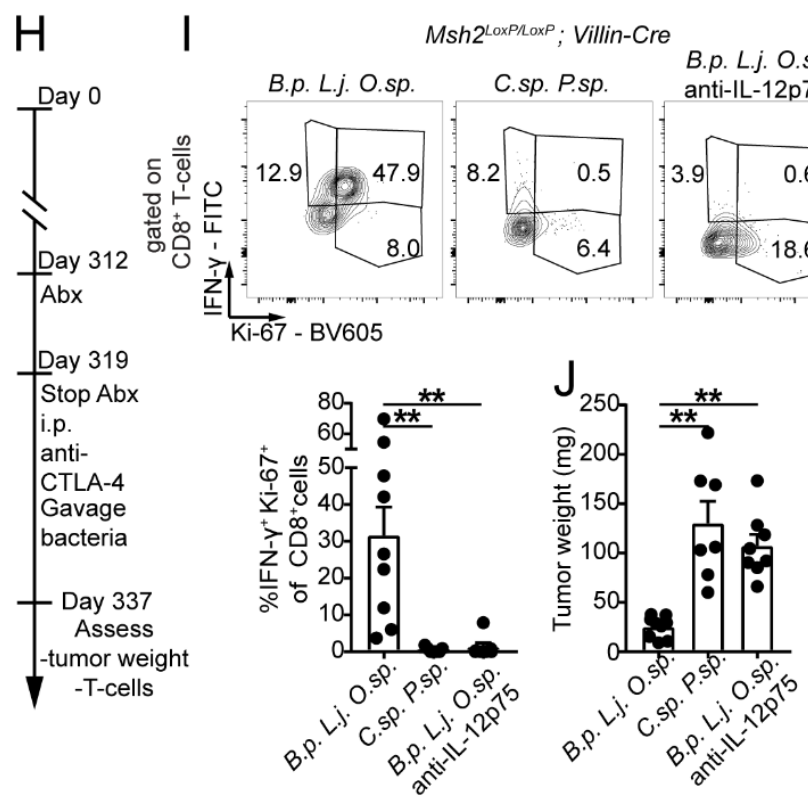

Figure 6: ICB therapy efficacy is CRC subtype dependent. (A, C, E and H) Schematic overview of the experimental setups to assess the effect of ICB-promoting bacteria in different subtypes of CRC. (B and D) Survival curve of isotype, anti-CTLA-4 or ICB-promoting (B.p. L.j. O.sp.) or control (C.sp. P.sp.) and anti-CTLA-4 co-treated $A p c^{2 l o x 14 /+} ; \mathrm{Kras}^{L S L-G 12 D /+} ; \mathrm{Fabpl}$ $\mathrm{Cre}$ animals. (F) Representative plots and quantification of intratumoral IFN- $\gamma^{+}, \mathrm{Ki}-67^{+}, \mathrm{CD}^{+}$

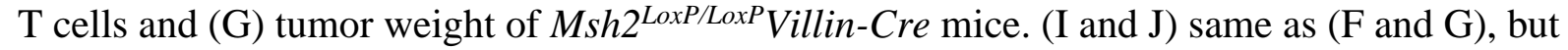
for bacteria, anti-CTLA-4 co-treated and anti-IL-12p75 co-treated mice as indicated. Data are mean \pm SEM and pooled from (B and F) five or (D and I) three individual experiments. (B) $n$ $=8-9,(\mathrm{D}) n=6-7,(\mathrm{~F}$ and $\mathrm{G}) n=10,(\mathrm{I}$ and $\mathrm{J}) n=7-9$ mice/group. $*, P<0.05 ; * *, P<0.01$. 